\title{
Deflection of Centrally Loaded Thin Circular Elastic Plates on Equally Spaced Point Supports
}

\author{
A. F. Kirstein, W. H. Pell,* R. M. Woolley, and L. J. Davis
}

(July 8, 1966)

\begin{abstract}
Bassali's general theory for the flexure of the thin circular elastic plate supported at an arbitrary number of points and subjected to transverse load over an eccentric circle is specialized to the case of a centrally loaded plate supported at points equally spaced on a circle concentric with the center. Simplified methods for approximating the results predicted by the more complicated theoretical expressions for deflection are presented along with the experimental results from 138 tests. Both the experimental results and the simplified equations are compared with the theory and agreement is found to be good.
\end{abstract}

Key Words: Bassali's theory, concentric loading, circular plates, deflection, elasticity, experimental, flexure, point supports, simplified approximate solutions, thin plates.

\section{Introduction}

The determination of the deflection of a centrally loaded circular plate supported at points equally spaced on a circle concentric with the center has long been an important structural analysis problem. In the past the analysis of this problem was usually limited by the assumption that the point supports were numerous enough to constitute a simple continuous line support. Nadai [1] ${ }^{1}$ presented a theory for the deformation of a circular plate supported at several points with central point load or uniform load which was an improvement in that it recognized the errors involved in the aforementioned assumption. Unfortunately, Nadai's point supports were located along the circumference of the plate. To some extent this limited the utility of the theory, as this method of support is unusually difficult to realize in practical structures.

More recently, Bassali [2] has given the solution of the problem of flexure of a thin circular elastic plate supported at an arbitrary number of points which may be located anywhere within the plate periphery, and loaded over a circular area lying anywhere within the boundary of the plate. Implicit in the work of Bassali is the solution of the problem of the centrally loaded plate supported at points equally spaced on a circle concentric with a central load. It may be noted that the theory accounts for the constraining effect of an annular region of the plate which overhangs the support circle and is otherwise free from restraint.

This paper deals with the specialization of that part of the Bassali theory necessary to solve the particular problem described above, and presents the rather tedious theoretical expressions for the deflection at the center of the plate and at a point midway between supports located along the support circle. Since these expressions require considerable effort to evaluate, simplified methods of approximating the center deflection may be desirable for design purposes. Therefore simplified expressions for center deflection, based on the results of the exact theory, are given. Experimental results are then compared with those obtained analytically, and good agreement between the two is found for the range of geometries tested.

*Present address: National Science Foundation, Room 305, Washington, D.C.

${ }^{1}$ Figures in brackets indicate the literature references at the end of this paper. 


\subsection{List of Symbols}

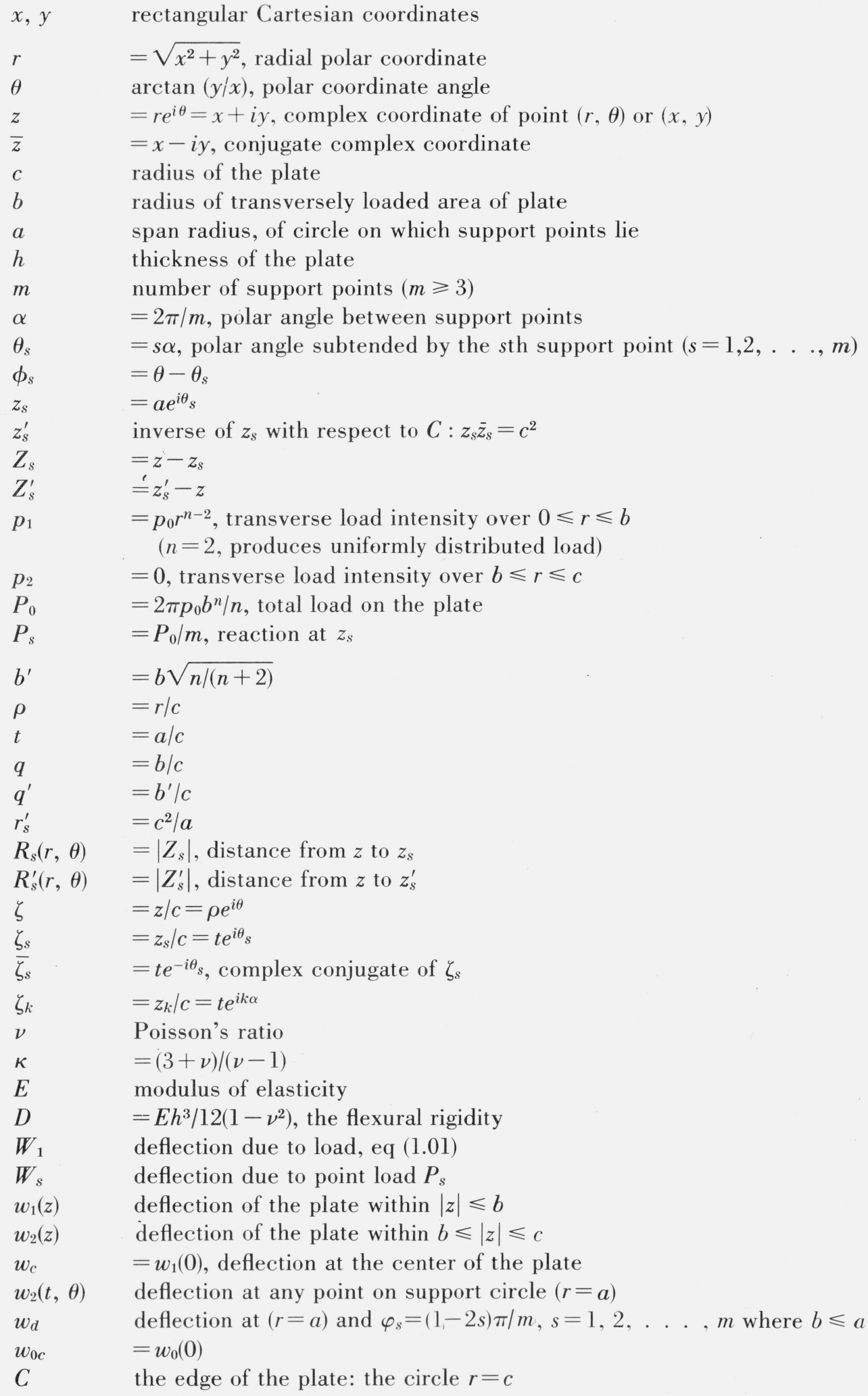


$\beta_{c} \quad$ dimensionless deflection function defined by eq (4.02)

$\beta_{c}^{\prime}$ dimensionless deflection function defined by eqs (4.16) and (4.18) dimensionless deflection function defined by eq (4.04)

$\psi$ dimensionless parameter defined by eq (4.17).

\section{Survey of Bassali's Procedure}

No extensive recapitulation of Bassali's paper [2] need be given here. Indeed, the theory is of such complexity that the mere presentation of the expressions for deflection is itself a lengthy process. It suffices to summarize the method used by Bassali and to note that his results were obtained through the use of the well-known theory of Muskhelishvili [3].

The center of a circular plate of radius $c$ is taken to be at the origin of a Cartesian coordinate system, and the central plane of the plate to lie in the $x y$ plane. The plate is assumed to carry a transverse load over the region ${ }^{2} 0 \leqslant r \leqslant b .^{3}$ The intensity of this load is

$$
p=p_{1}=p_{0} r^{n-2} \quad(n \geqslant 2)
$$

( $n=2$ produces uniform load). The transverse load intensity is $p=p_{2}=0$ over the remainder of the plate. The total load on the plate is $P_{0}=2 \pi p_{0} b^{n} / n$. The plate is taken to be supported at points equally spaced along the circle $r=a(b \leqslant a \leqslant c)$, viz, at $z_{s}=a e^{i s \alpha}(s=1,2, \ldots, m)$. From conditions of static equilibrium and symmetry the reactive force is the same at each support point $z_{s}$, and is $P_{s}=P_{0} / m$.

To obtain the deflection it is necessary to find the particular solutions for the plate equation, for eq (1.01), and for the point loads $P_{0} / m$. The former is easily seen by inspection to be

$$
W_{1}=\frac{p_{0}}{n^{2}(n+2)^{2} D}\left(Z_{0} \bar{Z}_{0}\right)^{\frac{n+2}{2}}
$$

It is well known from the theory of plates [4] that for the isolated point load $P_{s}$ at $z_{s}$

$$
W_{s}=\frac{P_{s}}{8 \pi D} R_{s}^{2} \ln R_{s}
$$

Bassali considers the plate to be comprised of two separate plates. One of these is defined by the loaded circular area $(0 \leqslant r \leqslant b)$, and the other is the annular plate $(b \leqslant r \leqslant c)$ with the point loads $P_{s}$. The solution of the equation for the plate deflection with the load $p_{1}$ is then the sum of eq (1.02) and the solution of the biharmonic equation; the solution for the annular plate is eq (1.03), summed over all of the point supports, plus the solution of the biharmonic equation. Boundary conditions for the free edge $r=c$, together with kinematic and dynamic conditions of continuity along $r=b[5]$ then make it possible to obtain the deflection, $w$, for any point on the plate.

\section{Deflection of the Circular Plate}

The deflection of the plate within the loaded region, $|z| \leqslant b$, obtained from Bassali's work is

$$
w_{1}=w_{0}+\frac{P_{0}}{8 \pi D}\left[\left(r^{2}+b^{\prime 2}\right) \ln q-\frac{1}{m} \sum_{s=1}^{m} R_{s}^{2} \ln \frac{R_{s}}{c}+\left(1-\frac{1}{n}\right)\left(r^{2}-b^{2}\right)+\frac{4\left(r^{n+2}-b^{n+2}\right)}{n(n+2)^{2} b^{n}}\right]
$$

\footnotetext{
${ }^{2}$ Bassali's solution is more general, in that, the loaded area and supports may be arbitrarily located anywhere within the plate periphery.

${ }^{3}$ For the reader's convenience a list of symbols is given in section 1.1 .
} 
while in the region $b \leqslant|z| \leqslant c$ it is

$$
w_{2}=w_{0}+\frac{P_{0}}{8 \pi D}\left[\left(r^{2}+b^{\prime 2}\right) \ln \rho-\frac{1}{m} \sum_{s=1}^{m} R_{s}^{2} \ln \frac{R_{s}}{c}\right]
$$

where

$$
w_{0}=\frac{P_{0}}{8 \pi m \kappa D}\left[\sum_{s=1}^{m} R^{2} \ln \frac{R_{s}^{\prime}}{r_{s}^{\prime}}+\left(\kappa^{2}-1\right) c^{2} \operatorname{Re}\left\{L_{m}(\zeta)\right\}+\frac{m\left(1-\rho^{2}\right)\left(a^{2}+\kappa b^{\prime 2}\right)}{\kappa+1}\right]+\gamma_{1} x+\gamma_{2} y+\gamma_{3}
$$

and

$$
\begin{aligned}
R_{s}^{2}(\rho, \theta) & =a^{2}\left(1+\frac{\rho^{2}}{t^{2}}-2 \frac{\rho}{t} \cos \varphi_{s}\right) \\
\frac{R_{s}^{2}}{c^{2}} & =\rho^{2}-2 \rho t \cos \varphi_{s}+t^{2} \\
\frac{R_{s}^{\prime 2}}{r_{s}^{\prime 2}} & =1-2 \rho t \cos \varphi_{s}+\rho^{2} t^{2} .
\end{aligned}
$$

The quantity $L_{m}(\zeta)$ which appears in eq (2.03) plays an important role in the deflection, and is defined by

$$
L_{m}(\zeta)=\sum_{s=1}^{m} \int_{0}^{1}\left(1-\bar{\zeta}_{s} \zeta u\right) \ln \left(1-\bar{\zeta}_{s} \zeta u\right) \frac{d u}{u}=\sum_{s=1}^{m}\left[-\varphi\left(\bar{\zeta}_{s} \zeta\right)+\bar{\zeta}_{s} \zeta+\left(1-\bar{\zeta}_{s} \zeta\right) \ln \left(1-\bar{\zeta}_{s} \zeta\right)\right]
$$

and where $\varphi\left(\bar{\zeta}_{s} \zeta\right)$ is defined as the dilogarithm $[6,7,8]$

$$
\varphi\left(\bar{\zeta}_{s} \zeta\right)=-\int_{0}^{\bar{\zeta}_{s} \zeta} \ln (1-\tau) \frac{d \tau}{\tau}, \quad\left|\bar{\zeta}_{s} \zeta\right|<1
$$

This function possesses the absolutely and uniformly convergent expansion [8]

$$
\varphi\left(\bar{\zeta}_{s} \zeta\right)=\sum_{n=1}^{\infty} \frac{\left(\bar{\zeta}_{s} \zeta\right)^{n}}{n^{2}}, \quad\left|\bar{\zeta}_{s} \zeta\right|<1
$$

Since $\bar{\zeta}_{s} \zeta=\rho t e^{i \varphi_{s}}$, eq (2.09) can be written as

$$
\varphi\left(\bar{\zeta}_{s} \zeta\right)=\sum_{n=1}^{\infty} \frac{(\rho t)^{n}}{n^{2}} e^{i n \varphi_{s}}
$$

and if $\zeta=\zeta_{k}, k=1,2, . ., m$, eq $(2.10)$ becomes

$$
\sum_{s=1}^{m} \varphi\left(\bar{\zeta}_{s} \zeta_{k}\right)=\frac{1}{m} \varphi\left(t^{2 m}\right)
$$

From eqs (2.07) and (2.10) the real part of $L_{m}(\zeta)$ can be written

$\operatorname{Re}\left\{L_{m}(\zeta)\right\}=-\sum_{n=1}^{\infty} \frac{(\rho t)^{n}}{n^{2}} \sum_{s=1}^{m} \cos n \varphi_{s}+\frac{1}{2} \sum_{s=1}^{m}\left(1-\rho t \cos \varphi_{s}\right) \ln \left(1-2 \rho t \cos \varphi_{s}+\rho^{2} t^{2}\right)$

$$
-\rho t \sum_{s=1}^{m} \sin \varphi_{s} \arctan \frac{\rho t \sin \varphi_{s}}{1-\rho t \cos \varphi_{s}}
$$


When $\zeta=\zeta_{k}=t e^{i k \alpha}$, eq $(2.12)$ becomes

$\operatorname{Re}\left\{L_{m}\left(\zeta_{k}\right)\right\}=-\frac{1}{m} \varphi\left(t^{2 m}\right)+\frac{1}{2} \sum_{s=1}^{m}\left(1-t^{2} \cos s \alpha\right) \ln \left(1-2 t^{2} \cos s \alpha+t^{4}\right)$

$$
-t^{2} \sum_{s=1}^{m} \sin s \alpha \arctan \frac{t^{2} \sin s \alpha}{1-t^{2} \cos s \alpha}
$$

For the purposes of this investigation it suffices to consider the case in which the support points lie outside the loaded area, i.e., $q \leqslant t$. Since the central plane of the undeformed plate is taken to lie in the $x y$ plane, $\gamma_{1}=\gamma_{2}=0$ in eq (2.03). Further, if the deflection is taken to vanish at a support point $z_{k}$, then

$$
w\left(z_{k}\right)=w_{2}\left(z_{k}\right)=0
$$

This, along with eqs (2.02) and (2.03) serve to determine $\gamma_{3}$ :

$$
\begin{aligned}
\gamma_{3}=\frac{P_{0}}{8 \pi \kappa D}[ & -\kappa\left(a^{2}+b^{\prime 2}\right) \ln t+\frac{\kappa}{m} \sum_{s=1}^{m} R_{s}^{2}\left(a, \theta_{k}\right) \ln \frac{R_{s}}{c}\left(a, \theta_{k}\right) \\
& \left.-\frac{1}{m} \sum_{s=1}^{m} R_{s}^{2}\left(a, \theta_{k}\right) \ln \frac{R_{s}^{\prime}\left(a, \theta_{k}\right)}{r_{s}^{\prime}}\left(a, \theta_{k}\right)-\frac{1}{m}\left(\kappa^{2}-1\right) c^{2} \operatorname{Re}\left\{L_{m}\left(\zeta_{k}\right)\right\}-\frac{\left(1-t^{2}\right)\left(a^{2}+\kappa b^{\prime 2}\right)}{\kappa+1}\right] .
\end{aligned}
$$

It is a notational convenience to define $A_{m}(t)$ and $B_{m}(t)$ as

$$
a^{2} A_{m}(t) \equiv \frac{1}{m} \sum_{s=1}^{m} R_{s}^{2}\left(a, \theta_{k}\right)\left[\kappa \ln \frac{R_{s}}{c}\left(a, \theta_{k}\right)-\ln \frac{R_{s}^{\prime}}{r_{s}^{\prime}}\left(\begin{array}{l}
\left(a, \theta_{k}\right) \\
\left(a, \theta_{k}\right)
\end{array}\right]\right.
$$

and

$$
m t^{2} B_{m}(t) \equiv \operatorname{Re}\left\{L_{m}\left(\zeta_{k}\right)\right\}
$$

Using eqs (2.04), (2.05), (2.06), and (2.13) the definitions of $A_{m}(t)$ and $B_{m}(t)$ may be written as follows:

$$
m A_{m}(t)=\sum_{s=1}^{m}(1-\cos s \alpha)\left[\kappa \ln 2 t^{2}(1-\cos s \alpha)-\ln \left(1-2 t^{2} \cos s \alpha+t^{4}\right)\right]
$$

$m t^{2} B_{m}(t)=\frac{1}{2} \sum_{s=1}^{m}\left(1-t^{2} \cos s \alpha\right) \ln \left(1-2 t^{2} \cos s \alpha+t^{4}\right)$

$$
-\frac{1}{m} \varphi\left(t^{2 m}\right)-t^{2} \sum_{s=1}^{m} \sin s \alpha \arctan \frac{t^{2} \sin s \alpha}{1-t^{2} \cos s \alpha} .
$$

Using these definitions, eq $(2.15)$ can be rewritten as ${ }^{4}$

$$
\gamma_{3}=\frac{P_{0}}{8 \pi \kappa D}\left[a^{2}\left\{A_{m}(t)+\left(1-\kappa^{2}\right) B_{m}(t)\right\}-\kappa\left(a^{2}+b^{\prime 2}\right) \ln t-\frac{\left(1-t^{2}\right)\left(a^{2}+\kappa b^{\prime 2}\right)}{\kappa+1}\right] .
$$

The deflection of the plate is given by eqs (2.01) and (2.02), where $w_{0}$ of eq (2.03) is obtained by inserting $\gamma_{1}=\gamma_{2}=0$ and the value of $\gamma_{3}$ given by eq (2.18).

The deflections at the center of the plate and along the support circle are of particular interest. At the center $r=\rho=0$, so that $R_{s}=a, R_{s} / c=t$, and $R_{s}^{\prime} / r_{s}^{\prime}=1$, hence eq $(2.01)$ yields

\footnotetext{
${ }^{4}$ Bassali's equation for $\gamma_{3}$ is in error in that he has $+\kappa\left(a^{2}-b^{\prime 2}\right) \ln t$ where eq $(2.18)$ has $-\kappa\left(a^{2}+b^{\prime 2}\right) \ln t$. His expression for $A_{m}(t)$ is also in error, since the first $t^{2}$ as well as the $s$ in both logarithmic terms of eq (2.16) are absent in Bassali's work.
} 


$$
w_{c}=w_{0 c}+\frac{P_{0}}{8 \pi D}\left[b^{\prime 2} \ln q-a^{2} \ln t-\left(1-\frac{1}{n}\right) b^{2}-\frac{4 b^{2}}{n(n+2)^{2}}\right]
$$

where

$$
w_{0 c}=\frac{P_{0}}{8 \pi \kappa D}\left[\frac{a^{2}+\kappa b^{\prime 2}}{\kappa+1}+a^{2}\left\{A_{m}(t)+\left(1-\kappa^{2}\right) B_{m}(t)\right\}-\kappa\left(a^{2}+b^{\prime 2}\right) \ln t-\frac{\left(1-t^{2}\right)\left(a^{2}+\kappa b^{\prime 2}\right)}{\kappa+1}\right]
$$

This latter results from noting that eq (2.07) gives $L_{m}(0)=0$ and substituting this, and $\gamma_{3}$ from eq (2.18) in eq (2.03). The replacement of $w_{0 c}$ in eq (2.19) by the right-hand member of eq (2.20) gives

$$
\begin{aligned}
& w_{c}=\frac{P_{0}}{8 \pi \kappa D}\left[a^{2}\left\{A_{m}(t)+\left(1-\kappa^{2}\right) B_{m}(t)\right\}-\kappa\left(2 a^{2}+b^{\prime 2}\right) \ln t\right. \\
& \left.\quad+\kappa b^{\prime 2} \ln q+\frac{t^{2}\left(a^{2}+\kappa b^{\prime 2}\right)}{\kappa+1}-\kappa b^{\prime 2} \frac{(n+3)}{(n+2)}\right] .
\end{aligned}
$$

On the support circle $\rho=t$ (or $r=a$ ), so that eqs (2.04) and (2.06) yield

$$
\begin{aligned}
R_{s}^{2}(t, \theta) & =2 a^{2}\left(1-\cos \varphi_{s}\right) \\
\frac{R_{s}^{\prime 2}}{r_{s}^{\prime 2}} & =1-2 t^{2} \cos \varphi_{s}+t^{4}
\end{aligned}
$$

from which it follows that on the support circle eqs (2.02) and (2.03) become

$$
w_{2}(t, \theta)=w_{0}(t, \theta)+\frac{P_{0} a^{2}}{8 \pi \kappa D m}\left\{m \kappa\left(1+\frac{q^{\prime 2}}{t^{2}}\right) \ln t-\kappa \sum_{s=1}^{m}\left(1-\cos \varphi_{s}\right) \ln \left[2 t^{2}\left(1-\cos \varphi_{s}\right)\right]\right\}
$$

and

$w_{0}(t, \theta)=\frac{P_{0} a^{2}}{8 \pi \kappa D m}\left[\sum_{s=1}^{m}\left(1-\cos \varphi_{s}\right) \ln \left(1-2 t^{2} \cos \varphi_{s}+t^{4}\right)+\frac{m\left(1-t^{2}\right)}{\kappa+1}\left(1+\frac{\kappa q^{\prime 2}}{t^{2}}\right)+\frac{\left(\kappa^{2}-1\right)}{t^{2}} \operatorname{Re}\left\{L_{m}\left(t e^{i \theta}\right)\right\}\right.$.

The value of $\gamma_{3}$ in the latter is

$$
\gamma_{3}=\frac{P_{0} a^{2}}{8 \pi \kappa D m}\left[-m \kappa\left(1+\frac{q^{\prime 2}}{t^{2}}\right) \ln t-\frac{m\left(1-t^{2}\right)}{\kappa+1}\left(1+\frac{\kappa q^{\prime 2}}{t^{2}}\right)+m\left\{A_{m}(t)+\left(1-\kappa^{2}\right) B_{m}(t)\right\}\right]
$$

which is a slightly different form of eq (2.18). These expressions combine to yield

$$
\begin{aligned}
w_{2}(t, \theta)=\frac{P_{0} a^{2}}{8 \pi m \kappa}\left\{\sum_{s=1}^{m}(1\right. & \left.-\cos \varphi_{s}\right) \ln \left(1-2 t^{2} \cos \varphi_{s}+t^{4}\right)+\frac{\left(\kappa^{2}-1\right)}{t^{2}} \operatorname{Re}\left\{L_{m}\left(t e^{i \theta}\right)\right\} \\
& \left.\quad-\kappa \sum_{s=1}^{m}\left(1-\cos \varphi_{s}\right) \ln \left[2 t^{2}\left(1-\cos \varphi_{s}\right)\right]+m\left[A_{m}(t)+\left(1-\kappa^{2}\right) B_{m}(t)\right]\right\} .
\end{aligned}
$$

It is of interest to note that $q^{\prime}$ does not appear in eq (2.24), so that the deflection along the support circle is independent of the radius of the central loaded area as long as $b \leqslant a$. 


\subsection{The Concentrated Central Load}

In this particularly important case the relevant form of $w$ is $w_{2}$ of eq (2.02), and $b=b^{\prime}=q=q^{\prime}=0$ must be inserted in this and in eq (2.03) to produce the expression for the deflection. The final result is

$$
w=w_{0}+\frac{P_{0}}{8 \pi D}\left(r^{2} \ln \rho-\frac{1}{m} \sum_{s=1}^{m} R_{s}^{2} \ln \frac{R_{s}}{c}\right)
$$

where

$$
w_{0}=\frac{P_{0}}{8 \pi m \kappa D}\left[\sum_{s=1}^{m} R_{s}^{2} \ln \frac{R_{s}^{\prime}}{r_{s}^{\prime}}+\left(\kappa^{2}-1\right) c^{2} \operatorname{Re}\left\{L_{m}(\zeta)\right\}+\frac{m\left(1-\rho^{2}\right) a^{2}}{\kappa+1}\right]+\gamma_{3}
$$

and

$$
\gamma_{3}=\frac{P_{0} a^{2}}{8 \pi \kappa D}\left[A_{m}(t)+\left(1-\kappa^{2}\right) B_{m}(t)-\kappa \ln t-\frac{\left(1-t^{2}\right)}{\kappa+1}\right] .
$$

For comparison with experiment it is necessary to have the deflection at the center of the plate. In this instance $\rho=0$, so that eqs (2.04), (2.06), and (2.12) yield

$$
R_{s}=a, \frac{R_{s}^{\prime}}{r_{s}^{\prime}}=1, \text { and } \operatorname{Re}\{L(0)\}=0
$$

Combining eqs (3.01), (3.02), and (3.03) for this case gives

$$
w_{c}=w(0)=\frac{P_{0} a^{2}}{8 \pi \kappa D}\left[A_{m}(t)+\left(1-\kappa^{2}\right) B_{m}(t)+\frac{t^{2}}{\kappa+1}-2 \kappa \ln t\right] .
$$

The configuration for which the supports are at the edge of the centrally loaded plate is also of interest. The expression for center deflection is obtained by setting $t=1$ in eq (3.04). The result is

$$
w_{c 1}=\frac{P_{0} a^{2}}{8 \pi \kappa D}\left[A_{m}(1)+\left(1-\kappa^{2}\right) B_{m}(1)+\frac{1}{\kappa+1}\right]
$$

where

$$
\begin{aligned}
& m A_{m}(1)=4(\kappa-1) \sum_{s=1}^{m} \sin ^{2} \frac{s \alpha}{2} \ln \left(2 \sin \frac{s \alpha}{2}\right) \\
& m B_{m}(1)=\frac{m A_{m}(1)}{2(\kappa-1)}-\frac{1}{2} \sum_{s=1}^{m}(\pi-s \alpha) \sin s \alpha-\frac{\pi^{2}}{6 m}
\end{aligned}
$$

The last term in eq (3.07) comes from the fact that $\varphi(1)=\frac{\pi^{2}}{6} \quad[6]$.

The case represented by eq (3.05) was considered by Nadai. In order to compare results 
with Nadai's work Bassali calculated $w_{c 1}$ for $m=3$ (using $\nu=1 / 4$ ) and obtained

$$
w_{c 1}=0.754 \frac{P_{0} a^{2}}{E h^{3}}
$$

which coincides with the value obtained by Nadai. ${ }^{5}$

\section{Simplified Approximation of the Deflection Formulas}

The deflection equations presented in the preceding section are of such complexity that it is a lengthy process to write the expressions for deflection at any point on the plate. Therefore, a more simple and direct way of presenting the theoretical equations is devised. To illustrate this, attention is directed to the expressions for deflections at two points: the center of the plate and the point on the support circle midway between adjacent supports.

Inspection of eqs (3.01), (3.02), and (3.03) shows that by the substitution of $D=E h^{3} / 12\left(1-\nu^{2}\right)$ the deflection for concentrated central loading can be written in the form $w=P_{0} a^{2} / E h^{3}$ times a dimensionless function of $\rho, \theta, t, \nu$, and $m$. In particular, for the center deflection eq (3.04) gives

$$
w_{c}=\beta_{c} \frac{P_{0} a^{2}}{E h^{3}}
$$

where the center deflection function is

$$
\beta_{c}=\frac{3\left(1-\nu^{2}\right)}{2 \pi \kappa}\left[A_{m}(t)+\left(1-\kappa^{2}\right) B_{m}(t)+\frac{t^{2}}{\kappa+1}-2 \kappa \ln t\right] .
$$

Similarly, the deflection at the point on the support circle midway between consecutive support points may be written

$$
w_{d}=\beta_{d} \frac{P_{0} a^{2}}{E h^{3}}
$$

where the deflection function is

$$
\begin{aligned}
\beta_{d}=\frac{3\left(1-\nu^{2}\right)}{2 \pi \kappa}\left[A_{m}(t)\right. & +\left(1-\kappa^{2}\right) B_{m}(t)+\frac{\left(\kappa^{2}-1\right)}{m t^{2}} \operatorname{Re}\left\{L_{m}\left(t e i \frac{\pi}{m}\right)\right\} \\
+ & \frac{1}{m} \sum_{s=1}^{m}\left\{1-\cos \frac{\pi}{m}(1-2 s)\right\} \ln \left\{1-2 t^{2} \cos \frac{\pi}{m}(1-2 s)+t^{4}\right\} \\
& \left.-\frac{\kappa}{m} \sum_{s=1}^{m}\left\{1-\cos \frac{\pi}{m}(1-2 s)\right\} \ln 2 t^{2}\left\{1-\cos \frac{\pi}{m}(1-2 s)\right\}\right] .
\end{aligned}
$$

This is obtained by inserting $\theta=2 \pi / m$ in eq $(2.24)$, i.e., the angular coordinate of the point on the support circle midway between $\theta=0$ and $\theta=2 \pi / m$.

\footnotetext{
${ }^{5}$ It should be noted that the erroneous term obtained by Bassali in $\gamma_{3}$ will have no effect on eq (3.05) since both that term and the correct one given in eq (2.18) vanish when $t=1$. Even though it is beyond the scope of interest of this paper, it is noted here that an error also exists in Bassali's expression for $\gamma_{3}$ for the case $q \geqslant t$. As in the case $q \leqslant t$ above, the erroneous term vanishes when $t=1$. Further, there is evidence that the error noted in Bassali's expression for $A_{m}(t)$ is merely typographical, since he gives the correct result in eqs (3.06) and (3.07).
} 


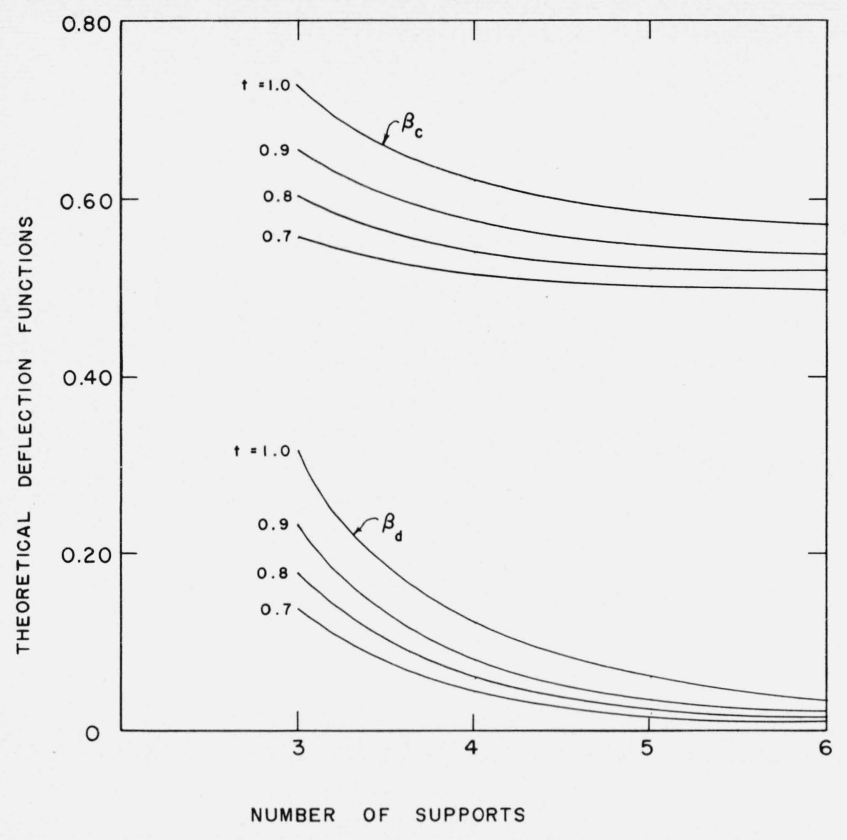

FIGURE 1. Relationship of theoretical deflection functions to number of supports.

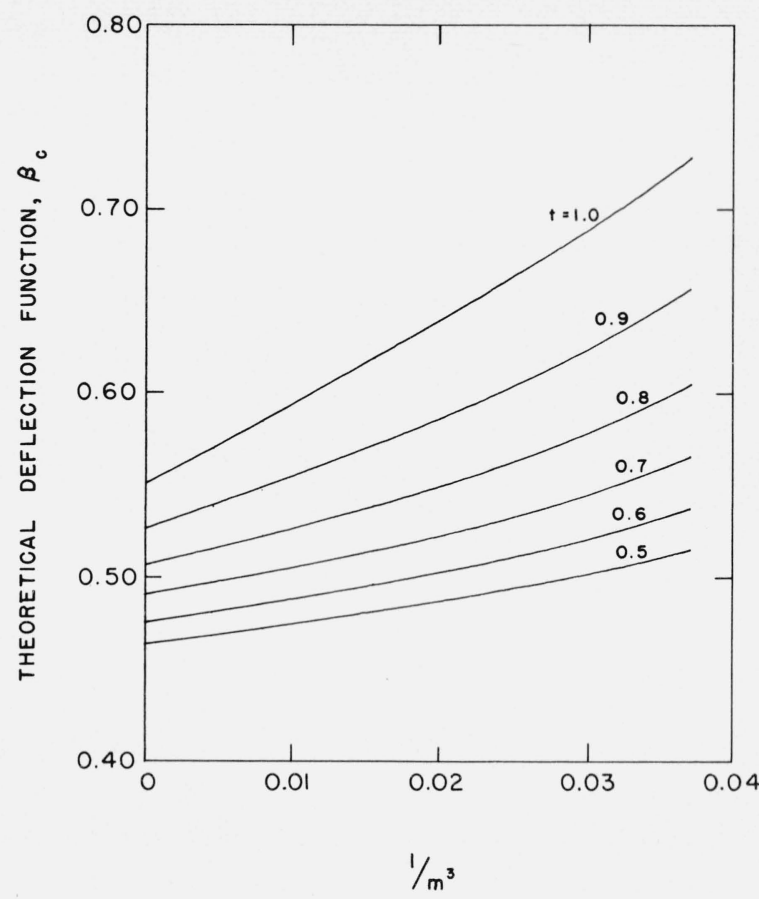

Figure 2. Approximation of $\beta_{\mathrm{c}}$ when $\mathrm{m} \rightarrow \infty$.

In figure $1 \beta_{c}$ and $\beta_{d}$ given by eqs (4.02) and (4.04), respectively, are plotted against $m$, with $t$ as a parameter. The value of $\nu$ was taken to be 0.3 . Examination of this figure shows that the deflection functions change rapidly with $m$ for $m<6$. However, very little change in the deflection functions for $m>6$ was indicated by eqs (4.02) and (4.04). Thus the error involved in assuming (in accord with the practice mentioned earlier) that multiple point supports constitute continuous line support changes rapidly as $m$ becomes smaller than 6 , but changes very little for $m>6$. As illustrated in figure $1 \beta_{d}$ approaches zero as $m$ increases. Further, it is expected that when $m$ becomes sufficiently large the support circle will tend to act as a continuous line support.

Unfortunately the deflection function, $\beta_{c}$, can not be evaluated directly from eq (4.02) by inserting $m=\infty$ to represent the case of the simple continuous line support. However, the solution of eq (4.02) for this case can be approximated in the following manner. Theoretical values of $\beta_{c}$ were computed from eq (4.02) for $m=3,4,5$, and 6 with $t$ and $\nu$ as parameters. Figure 2 presents typical results in the form of a family of curves for $\nu=0.3$ with $t$ as a parameter. For each $t$ these curves are assumed to be of the form

$$
\beta_{c}=a_{0}+a_{1} m^{-3}+a_{2} m^{-6}
$$

where $a_{0}, a_{1}$, and $a_{2}$ are constants. The method of least squares was used to evaluate the constants. To illustrate, when $t=1$ and $\nu=0.3$ eq (4.05) becomes

$$
\beta_{c}=0.551+4.172 m^{-3}+17.493 m^{-6} .
$$

Equation (4.06) is a mathematical expression of the $t=1$ curve shown in figure 2. Thus at $m=\infty$, $\beta_{c}=0.551$, and from eq (4.01) the center deflection becomes

$$
w_{c}=0.551 \frac{P_{0} a^{2}}{E h^{3}} .
$$


Comparing these results with those of the well-known expression for deflection [4]

$$
w_{\max }=\frac{(3+\nu) P a^{2}}{16 \pi(1+\nu) D}
$$

and supplying $D=E h^{3} / 12\left(1-\nu^{2}\right)$ where $\nu=0.3$, the center deflection becomes

$$
w_{\max }=0.551 \frac{P_{0} a^{2}}{E h^{3}} .
$$

The agreement of eqs (4.07) and (4.09) lends credence to the assumptions used to develop the approximate solution illustrated by eq (4.05).

Since it is evident that the evaluation of the theoretical deflection functions presented earlier requires considerable effort, it follows that the equations may have limited practical usefulness. It was for this reason that a simplified method of approximating the more important deflection function, $\beta_{c}$, (center deflection) was devised.

The development of the simplified approximate solution was accomplished through a trial process of plotting and comparing the theoretical values of $\beta_{c}$ against functions of the variables $t, m$, and $\nu$ in eq (4.02) and applying the least squares method of fitting curves to the theoretical results. Various forms of equations were tried, including the exponential and power forms. However, the most satisfactory was the consecutive applications of linear least squares in the following sequence: for each value of $m$ and $\nu$ an equation was written in the form

$$
\beta_{c}=a_{0}+b_{0} t^{3}
$$

where symbols $a$ and $b$ with subscripts do not refer to the previously defined radii, $a$, and $b$; then equations were written for $a_{0}$ and $b_{0}$ for each value of $m$ in the forms

$$
\begin{aligned}
& a_{0}=a_{1}+b_{1}\left(1-\nu^{2}\right) \\
& b_{0}=a_{2}+b_{2}\left(1-\nu^{2}\right)
\end{aligned}
$$

finally, equations were written for the symbols in eq (4.11) in the forms

$$
\begin{aligned}
& a_{1}=a_{3}+b_{3} m^{-3} \\
& b_{1}=a_{4}+b_{4} m^{-3} \\
& a_{2}=a_{5}+b_{5} m^{-3} \\
& b_{2}=a_{6}+b_{6} m^{-3} .
\end{aligned}
$$

Combining this group of linear equations and substituting them into eq (4.10) gave

$$
\beta_{c}=a_{3}+b_{3} m^{-3}+\left(a_{4}+b_{4} m^{-3}\right)\left(1-\nu^{2}\right)+\left[a_{5}+b_{5} m^{-3}+\left(a_{6}+b_{6} m^{-3}\right)\left(1-\nu^{2}\right)\right] t^{3}
$$

or

$$
\begin{aligned}
\beta_{c}=-0.0642-2.1900 m^{-3}+ & \left(0.5687+3.2542 m^{-3}\right)\left(1-\nu^{2}\right) \\
+ & {\left[-0.3793+11.0513 m^{-3}+\left(0.5223-7.8535 m^{-3}\right)\left(1-\nu^{2}\right)\right] t^{3} . }
\end{aligned}
$$


Since the simplified method of computing $\beta_{c}$ given in eq (4.14) was developed through the curve fitting process described above, this equation reflects the arbitrarily selected limitations of the parameters. These limitations were as follows:

$$
0.25 \leqslant \nu \leqslant 0.33 \quad 0.50 \leqslant t \leqslant 1.00 \quad m=3,4,5,6, \text { and } \infty .
$$

The largest disparity between the results from eqs (4.14) and $(4.02)^{6}$ computed within the above stated limitations was 1.26 percent.

It is interesting to compare the expressions for center deflection when the load is applied uniformly $(n=2)$ to the plate through a centrally located circular area described by radius $b$ and for center deflection when $b$ is permitted to shrink to zero. Making use of the definition of a deflection function, $\beta$, eq (2.21) can be written as

$$
w_{c}=\beta_{c}^{\prime} \frac{P_{0} a^{2}}{E h^{3}}
$$

where

$$
\beta_{c}^{\prime}=\frac{3\left(1-\nu^{2}\right)}{2 \pi \kappa}\left[A_{m}(t)+\left(1-\kappa^{2}\right) B_{m}(t)+\frac{\kappa q^{2}}{2 t^{2}} \ln q+\frac{t^{2}}{\kappa+1}+\frac{\kappa q^{2}}{2(\kappa+1)}-\frac{\kappa q^{2}}{2 t^{2}} \frac{5}{4}-2 \kappa \ln t-\frac{\kappa q^{2}}{2 t^{2}} \ln t\right]
$$

$w_{c}$ and $\beta_{c}$ for the concentrated load at the center of the plate are given by eqs (4.01) and (4.02), respectively. By taking the difference between eqs (4.16) and (4.02) all of the terms in eq (4.16) containing $q$ can be collected into a separate expression and written as

$$
\psi=\frac{3\left(1-\nu^{2}\right)}{4 \pi} \frac{q^{2}}{t^{2}}\left[\frac{t^{2}}{\kappa+1}+\ln \frac{q}{t}-\frac{5}{4}\right]
$$

when $b \leqslant a$. This maneuver makes it possible to express $\beta_{c}^{\prime}$ as

$$
\beta_{c}^{\prime}=\beta_{c}+\psi
$$

but what is more important, it presents an opportunity to extend the utility of the simplified approximate solution for $\beta_{c}$ given in eq (4.14) to approximate the value of $\beta_{c}^{\prime}$ within the stated limitations of eq (4.14).

\section{Test Specimens}

A total of eight thin plate specimens were made for use in the experimental portion of this investigation. All of these flat circular specimens were made from bare sheets of 7075-T6 aluminum alloy. The modulus of elasticity for each sheet of aluminum was determined from tests of four tensile coupons. In all cases the direction of roll of the sheets was noted and two tensile specimens were oriented parallel and two perpendicular to the direction of roll. There were only small random differences between the moduli of elasticity with respect to the direction of roll. Therefore the average of the moduli parallel and perpendicular to the direction of roll of the sheet was selected to represent the moduli of elasticity of the plate specimens fabricated from the sheet. The physical properties of the plates are given in table 1. For computational purposes the Poisson's ratio for all of the specimens was assumed to be 0.3 .

${ }^{6}$ Computations of deflection for $m=\infty$ were based on the approximation illustrated by eq $(4.05)$. 
TABLE 1. Physical properties of plate specimens

\begin{tabular}{|c|c|c|c|}
\hline Specimen & $\begin{array}{c}\text { Thickness } \\
h\end{array}$ & $\underset{c}{\text { Radius }}$ & $\begin{array}{c}\text { Modulus } \\
\text { of } \\
\text { elasticity } \\
E\end{array}$ \\
\hline & & in & psi \\
\hline & 0.125 & 5.125 & $10.0 \times 10^{6}$ \\
\hline & .125 & 4.625 & $10.0 \times 10^{6}$ \\
\hline C... & .125 & 4.375 & $10.0 \times 10^{6}$ \\
\hline & .161 & 5.125 & $10.8 \times 10^{6}$ \\
\hline E. & .161 & 4.625 & $10.8 \times 10^{6}$ \\
\hline $\mathrm{F}$. & .161 & 4.375 & $10.8 \times 10^{6}$ \\
\hline G.. & .250 & 5.125 & $9.2 \times 10^{6}$ \\
\hline H... & 132 & 5.125 & $10.0 \times 10^{6}$ \\
\hline
\end{tabular}

The direction of roll was also noted on the plate specimens, and made to correspond to the $0^{\circ}$ to $180^{\circ}$ orientation of the plates. The dimensions of the specimens are given in table 1. Further examination of table 1 will reveal that Specimens A, B, and C were fabricated from the same 0.125 in. thick sheet, and Specimens D, E, and F were fabricated from a sheet 0.161 in. thick. Specimens $\mathrm{G}$ and $\mathrm{H}$ were fabricated from separate sheets of $0.250 \mathrm{in}$. and $0.132 \mathrm{in}$. thicknesses, respectively.

\section{Test Methods}

The specimens were supported by $1 / 4$ in. diam threaded studs having a machine finished spherical shape on the end that bore against the specimens. These supports were equipped with lock nuts and inserted into tapped holes in the steel base block shown in figure 3. This base was $185 / 8$ in. square and $5 \frac{3}{8}$ in. thick with a $3 \frac{3}{4}$ in. diam center hole. Positioning the support studs in this base made it possible to orient the supports to close tolerances for the cases of 3 , 4 , and 5 equally spaced supports at the span radii listed in table 2.

Deflection measurements were made with dial gages having a least division of 0.0001 in. These gages were mounted on the heavy steel base block described above using it as a datum plane.

Two different methods of applying load to the specimens were used. The diagrammetric sketches shown in figure 3 depict these methods. The dead weight loading technique was found to be the most convenient to use. It was necessary to drill and tap a $1 / 4-20$ hole in the center of seven of the eight specimens to facilitate load application. A small eye bolt was screwed into this hole and locked into position to receive the loading pan. It was felt that this small discontinuity would not seriously effect the load-deflection characteristics of the specimens, and the

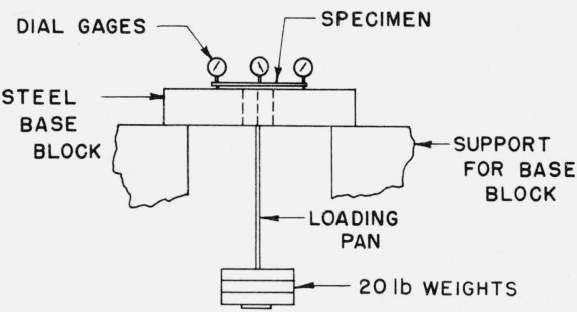

(a)

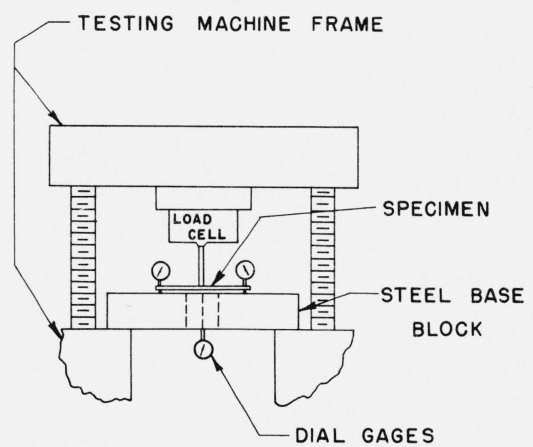

(b)

FIGURE 3. Methods used to apply load to test specimens.

3a. Load applied by dead weight.

3b. Load applied by testing machine. 
advantage of conveniently applying known load increments would outweigh the disadvantage of loading through the screw threads. To verify this assumption Specimen $\mathrm{H}$ was made as a companion to Specimens A, D, and G except that it did not have the small tapped center hole and was loaded in a testing machine frame through a 600 -pound capacity elastic load measuring device as shown in figure 3 .

The test procedure in both methods of load application was the same. The load was applied in 20-pound increments with the center deflection and deflection midway between supports at $r=a$ being recorded for each increment of load.

TABLE 2. Span radii of equally spaced point supports

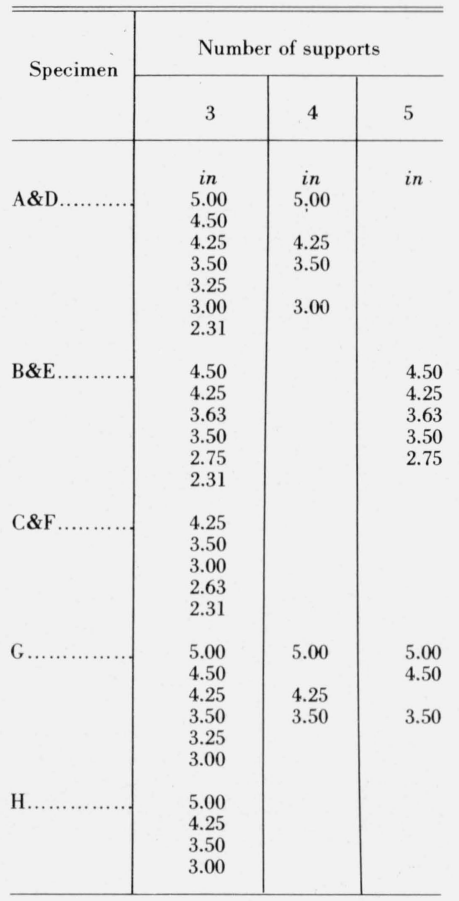

\section{Experimental Results}

The experimental deflection data of the plate tests are given in table 3 as the deflection functions, $\beta_{c}$ and $\beta_{d}$, defined by eqs (4.02) and (4.04), respectively. These experimental deflection functions were computed from eqs (4.01) and (4.03) using the deflection sensitivity, $w / P_{0}$, as determined from the test data. Typical load-deflection data are shown in figure 4. The reciprocals of the slopes of these curves are the deflection sensitivities in inches per pound. As mentioned previously the plates were tested on 3,4 , and 5 equally spaced point supports with the range of span radii as shown in table 2 . Table 3 is arranged to show the support conditions as well as the experimental results for all of the plate specimens.

Comparisons of the experimental and theoretical deflection functions are given in figure 5 . This figure presents three coordinate systems representing the cases $m=3,4$, and 5 . The deflection functions are shown as the ordinate and $t$, is the abscissa. The theoretical relationships between $\beta$ and $t$ from eqs (4.02) and (4.04) for a Poisson's ratio of $\nu=0.3$ are represented by the solid lines. As indicated in the figure there was good agreement between the experimental and theoretical results. 
TABLE 3. Experimental deflection functions, $\beta_{\mathrm{c}}$ and $\beta_{\mathrm{d}}$

\begin{tabular}{|c|c|c|c|c|c|c|c|c|}
\hline \multirow{3}{*}{$\begin{array}{c}\text { Speci- } \\
\text { men }\end{array}$} & \multirow{3}{*}{$\begin{array}{l}\text { Span } \\
\text { radii } \\
\quad a\end{array}$} & \multirow{3}{*}{$\begin{array}{c}\text { Ratio of } \\
\text { span to speci- } \\
\text { men radii } \\
t\end{array}$} & \multicolumn{6}{|c|}{ Number of supports } \\
\hline & & & \multicolumn{2}{|c|}{3} & \multicolumn{2}{|c|}{4} & \multicolumn{2}{|c|}{5} \\
\hline & & & $\beta_{c}$ & $\beta_{d}$ & $\beta_{c}$ & $\beta_{d}$ & $\beta_{c}$ & $\beta_{d}$ \\
\hline A & $\begin{array}{l}\text { in } \\
5.00\end{array}$ & 0.9756 & 0.6763 & & 0.5447 & 0.1104 & & \\
\hline & 5.00 & .9756 & .6771 & 0.3007 & .5486 & .1126 & & \\
\hline & 5.00 & .9756 & .6834 & .3382 & .5423 & .1087 & & ......... \\
\hline & 5.00 & .9756 & & & .5496 & .1079 & & \\
\hline & 4.50 & .8780 & .6468 & .2371 & & & & \\
\hline & 4.50 & .8780 & $\begin{array}{l}.6546 \\
.6010\end{array}$ & .2581 & .4935 & .0649 & & \\
\hline & $\begin{array}{l}4.25 \\
4.25\end{array}$ & $\begin{array}{l}.8292 \\
.8292\end{array}$ & $\begin{array}{l}.6010 \\
.5891\end{array}$ & .1967 & .4908 & .0658 & & $\cdots$ \\
\hline & 4.25 & .8292 & & & .4986 & .0693 & & w.... \\
\hline & 3.50 & .6829 & .5308 & .1328 & .4675 & .0427 & & ….... \\
\hline & 3.50 & .6829 & .5308 & .1366 & .4739 & .0419 & & ............. \\
\hline & 3.25 & .6341 & .5325 & .1387 & & & & ........... \\
\hline & 3.25 & .6341 & .5103 & .1239 & & & & \\
\hline & 3.00 & .5854 & .5013 & .1085 & .4516 & .0430 & & .......... \\
\hline & 3.00 & .5854 & .4947 & .1085 & .4531 & .0439 & & ........... \\
\hline & 2.31 & .4512 & .4765 & .1095 & & & & .............. \\
\hline & 2.31 & .4512 & .4761 & .1095 & & & & ................. \\
\hline B & 4.50 & .9730 & .7163 & .2940 & & & 0.5252 & 0.0530 \\
\hline & 4.50 & .9730 & & & & & .5342 & .0543 \\
\hline & 4.25 & .9189 & .6691 & .2465 & & & .4954 & .0408 \\
\hline & 4.25 & .9189 & & & & & .4954 & .0411 \\
\hline & 3.63 & .7838 & .5573 & .1725 & & & .4804 & .0294 \\
\hline & 3.63 & .7838 & .5543 & .1722 & & & .4754 & .0290 \\
\hline & 3.50 & .7568 & .5420 & .1631 & $\cdots$ & $\cdots$ & .4616 & .0258 \\
\hline & 3.50 & .7568 & .5451 & .1634 & & ..... & .4613 & .0277 \\
\hline & 2.75 & .5946 & .5035 & .1247 & 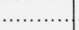 & $\ldots$. & .4467 & .0263 \\
\hline & 2.75 & .5946 & .5061 & .1139 & & $\ldots$ & .4480 & .0258 \\
\hline & 2.31 & .5000 & .4710 & .1095 & ........ & $\ldots \ldots$ & ......... & $\therefore \ldots \ldots \ldots \ldots$ \\
\hline & 2.31 & .5000 & .4819 & .1062 & & & & …............. \\
\hline C & 4.25 & .9714 & .6627 & .2735 & & & & \\
\hline & 3.50 & .8000 & .5547 & .1788 & & & & ....... \\
\hline & 3.50 & .8000 & .5531 & .1796 & & & & .......... \\
\hline & 3.00 & .6857 & .5197 & .1440 & & & & ......... \\
\hline & 3.00 & .6857 & .5186 & .1451 & & & & ......... \\
\hline & 2.63 & .6000 & .5113 & .1267 & & & & .......... \\
\hline & 2.63 & .6000 & .5101 & .1278 & & & & ......... \\
\hline & 2.31 & .5286 & .4958 & .1267 & & & & ......... \\
\hline & 2.31 & .5286 & .4867 & .1267 & & & & \\
\hline D & 5.00 & 0.9756 & 0.7645 & 0.3245 & 0.6296 & 0.1262 & & ........ \\
\hline & 5.00 & $\begin{array}{l}.9756 \\
9756\end{array}$ & ( & ( & $\begin{array}{l}.6256 \\
6256\end{array}$ & .1287 & & ......... \\
\hline & $\begin{array}{l}5.00 \\
4.50\end{array}$ & $\begin{array}{l}.9756 \\
.8780\end{array}$ & .7079 & .2506 & .6256 & .1287 & & ......... \\
\hline & 4.25 & .8292 & .6472 & .2202 & .5738 & .0774 & & …..... \\
\hline & 4.25 & .8292 & .6455 & .2195 & .5609 & .0779 & & , …...... \\
\hline & 3.50 & .6829 & .5888 & .1586 & .5299 & .0534 & & …..... \\
\hline & 3.50 & .6829 & .5873 & .1579 & .5303 & .0552 & & .......... \\
\hline & 3.25 & .6341 & .5654 & .1532 & & - & & $\ldots \ldots$. \\
\hline & 3.25 & .6341 & .5842 & .1553 & & & & ......... \\
\hline & 3.00 & .5854 & .5574 & .1322 & .5193 & .0496 & & .......... \\
\hline & 3.00 & .5854 & .5534 & .1347 & .5258 & .0551 & & .......... \\
\hline & 2.31 & .4512 & .5293 & .1114 & ... & 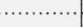 & ….... & ............ \\
\hline & 2.31 & .4512 & .5304 & .1161 & & & & ……… \\
\hline $\mathrm{E}$ & 4.50 & .9730 & .7304 & .3339 & & & 0.5957 & 0.0657 \\
\hline & 4.50 & .9730 & .7419 & .3388 & & & .6037 & .0661 \\
\hline & 4.25 & .9189 & .7021 & .2731 & & & .5783 & .0507 \\
\hline & 4.25 & .9189 & .7009 & .2741 & & & .5756 & .0494 \\
\hline & 3.63 & .7838 & .6294 & .2018 & & . & .5546 & .0343 \\
\hline & 3.63 & .7838 & .6328 & .2004 & & & .5443 & .0343 \\
\hline & 3.50 & .7568 & .6157 & .1840 & & & .5307 & .0357 \\
\hline & 3.50 & .7568 & .6090 & .1840 & & & .5399 & .0346 \\
\hline & 2.75 & .5946 & .5650 & .1401 & & & .5120 & .0387 \\
\hline & 2.75 & .5946 & .5668 & .1377 & & & .5191 & .0393 \\
\hline & 2.31 & .5000 & .5582 & .1308 & & & & ........ \\
\hline & 2.31 & .5000 & .5491 & .1313 & & & & .......... \\
\hline $\mathrm{F}$ & 4.25 & .9714 & .7156 & .3252 & & & & \\
\hline & 4.25 & .9714 & .7144 & .3252 & & & & ....... \\
\hline & 3.50 & .8000 & .6083 & .2031 & & & & $\ldots \ldots$ \\
\hline & 3.50 & .8000 & .6204 & .2046 & & & & ……….. \\
\hline & 3.00 & .6857 & .5774 & .1573 & & & & ….......... \\
\hline & 3.00 & .6857 & .5764 & .1573 & & & & ............ \\
\hline & 2.63 & .6000 & .5486 & .1372 & & & . & (........... \\
\hline & 2.63 & .6000 & .5579 & .1431 & & & & ............ \\
\hline & 2.31 & .5286 & .5280 & .1446 & & & & ……… \\
\hline & 2.31 & .5286 & .5268 & .1490 & & & & \\
\hline
\end{tabular}


TABLE 3. Experimental deflection functions, $\beta_{\mathrm{c}}$ and $\beta_{\mathrm{d}}-$ Continued

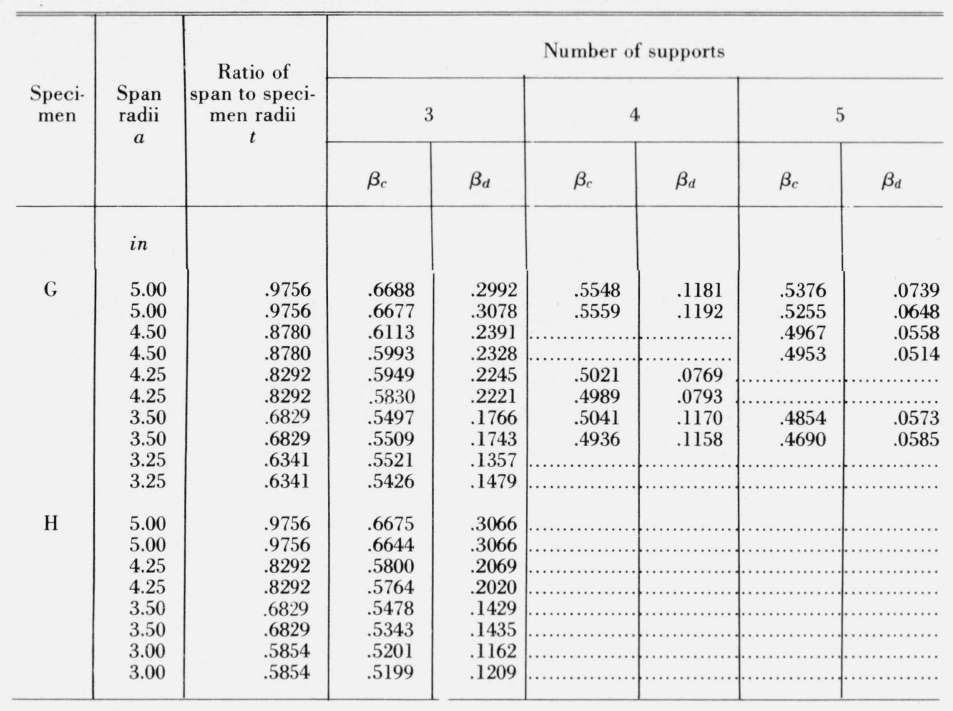

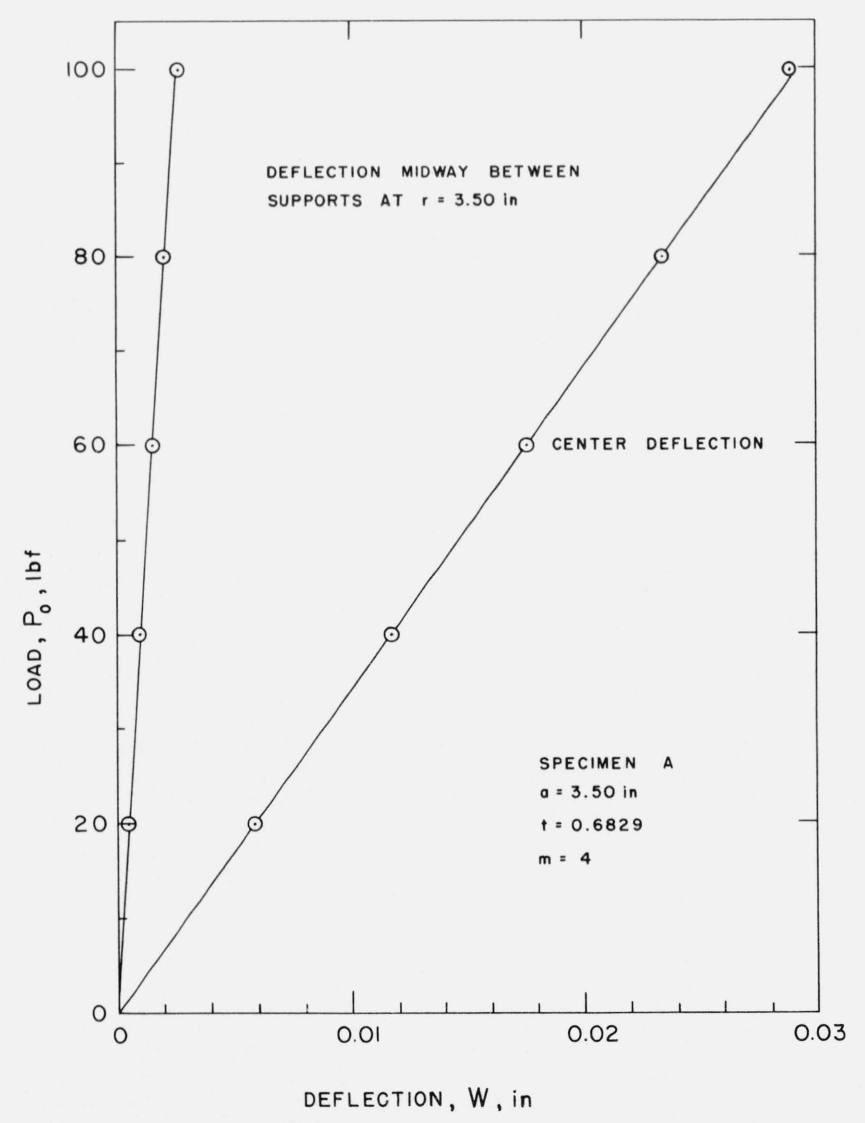

Figure 4. Typical load versus deflection test data. 


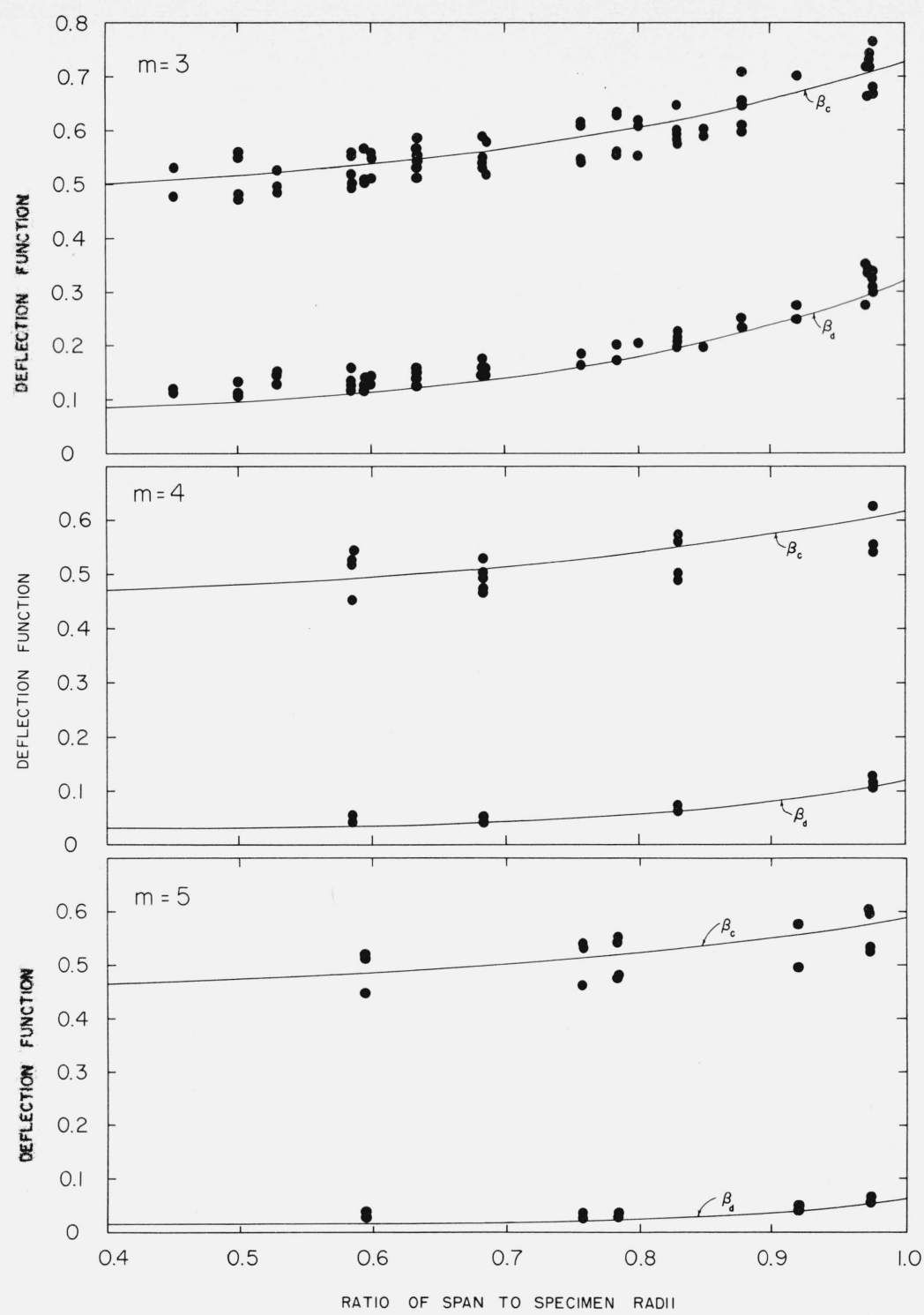

Figure 5. Comparison of theoretical and experimental deflection functions.

Since the maximum deflection, represented by $\beta_{c}$, is usually of more interest, a direct comparison of the experimental and theoretical $\beta_{c}$ values is made in figure 6 with an indication of the order of magnitude of error involved.

All of the experimental data represented by circular symbols in figure 6 is presented in table 3 , and represents the full range of this experimental investigation for $m=3$. The solid circular symbols labeled A and $\mathrm{H}$ were identified to show the effect of a tapped center hole in Specimen A on the load-deflection characteristics as compared with Specimen H which did not have a center hole and was approximately the same thickness. It can be seen from the data that the effect was so small that the disparity of experimental points from all of the other specimens was greater than that for companion Specimens A and H. The triangular symbols in figure 6 represent results reported by Nadai [1] on three very thin glass plates. 
FIGURE 6. Experimental and theoretical center deflection functions.

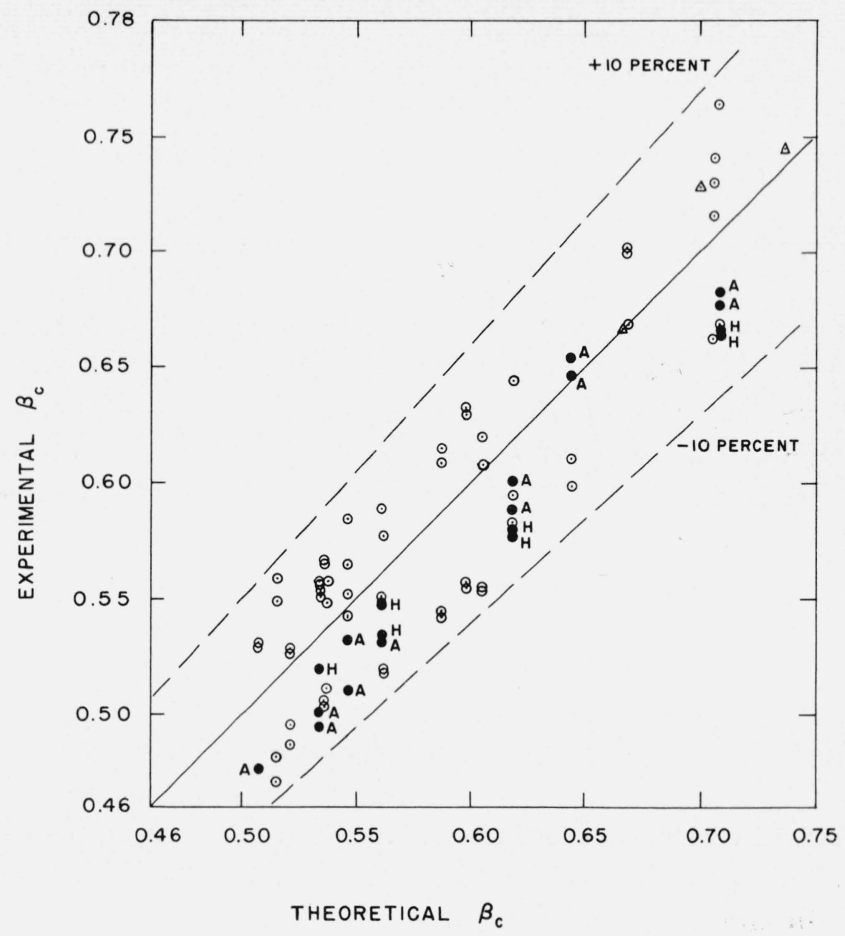

8. Discussion

Bassali obtained the solution for the problem of flexure of a thin circular elastic plate supported at several arbitrarily located points (the boundary of the plate was free), and transversely loaded over an eccentrically located circular area (the load was symmetrically distributed with respect to the center of the loaded area). The general solution provided by Bassali was specialized for this investigation of the problem of the concentrically loaded plate supported at points equally spaced on a circle concentric with the central load, and located in the unloaded area, i.e., $q \leqslant t$.

As noted previously, two expressions for deflection are required; $w_{1}$ being the deflection of the plate within the loaded region $(r \leqslant b)$, and $w_{2}$ the deflection in the unloaded region $(r \geqslant b)$. The deflection of the plate obtained from Bassali's work is given in eqs (2.01) and (2.02), where $w_{0}$ is obtained from eq (2.03) by inserting $\gamma_{1}=\gamma_{2}=0$ and the value of $\gamma_{3}$ given by eq (2.18). It was noted that certain errors were apparent in Bassali's work (see footnotes 4 and 5), but these were of a minor nature and stand corrected in eqs (2.16) and (2.18) of this paper for the case $q \leqslant t$.

Bassali's work was specialized further to examine the case of the concentrated central load for the purpose of comparing his theory with the experimental results from 138 tests presented herein. It was found convenient to compare the measured deflections at the center and at a point midway between supports on the support circle with the theory in terms of the dimensionless deflection functions, $\beta_{c}$ and $\beta_{d}$, given in eqs (4.02) and (4.04), respectively. This comparison was made in figures 5 and 6 , and good agreement between theoretical and experimental results was apparent.

It is of some importance to note from figure 5 that both the theoretical and experimental results indicate that the deflection functions decrease as the annular portion of the plate overhanging the support circle increases. It is of further interest to note from figures 1 and 2 that this constraining effect decreases as the number of supports, $m$, increases, but does not vanish for $\beta_{c}$ when $m$ becomes sufficiently large to produce a support condition equivalent to that of a simple continuous line support. From this it would appear desirable to consider the effect of the overhanging portion of the plate in the design and analysis of practical structures of this type for both conditions of support discussed above. 
Bassali's expressions for deflection are of such complexity that they are probably unsuitable for design purposes, and there is no direct solution available from his work for the case of simple continuous line support. However, the family of curves shown in figure 2 along with the apparent $y$-intercepts suggested the simple method of approximating $\beta_{c}$ for the simple support condition as illustrated by eq (4.05). The result of this approximation given in eq (4.07), where $t=1$, agreed favorably with that from the classical elementary theory for the case of simple support at the boundary of the plate. Thus, the assumptions used to develop the approximate solution given in eqs (4.05) and (4.06) were substantiated.

Further interest in simplified design equations produced the expression for approximating the theoretical center deflection function, $\beta_{c}$. The development of this expression, which approximates the results of the theoretical expression in eq (4.02), is described by eqs (4.10-4.13), and presented in eq (4.14). As stated previously the largest difference between the results of the approximate solution and the theoretical solution computed within the limitations inherent in eq (4.14) was 1.26 percent. Since such good agreement exists between the approximate and theoretical results, eq (4.14) should prove useful for design purposes. It should be noted here that setting $m^{-3}=0$ in eq (4.14) produces the approximate expression for $\beta_{c}$ when there is a condition of simple continuous line support at $t$. This case was discussed earlier and illustrated in eq (4.05).

A comparison of expressions for the theoretical center deflection functions, $\beta_{c}$ and $\beta^{\prime}{ }_{c}$, given in eqs (4.02) and (4.16), respectively, indicated that the approximate solution for $\beta_{c}$ given in eq (4.14) could be modified to approximate $\beta^{\prime}{ }_{c}$ of eq (4.16) as indicated in eqs (4.17) and (4.18). Since $\psi$ is obtained'directly from the theory, no further disparity is expected between the theoretical and the approximate $\beta^{\prime}{ }_{c}$ values.

\section{Conclusions}

1. In view of the good agreement between the theoretical and experimental results from the 138 tests reported herein, Bassali's theory adequately predicts the deflection of the plates over the range of geometries investigated.

2. Both the theoretical and experimental results show that the deflection of the plate within the support circle is constrained by the annular portion of the plate which overhangs the support circle. This constraint is found to decrease as the overhang is decreased, and to increase as the number of supports are decreased. Since this constraining effect is not negligible for a plate overhanging a support circle containing many supports, it is an important consideration in the design and analysis of thin plate structures, and especially so when the number of supports are fewer than six.

3. The simplified equations for deflection functions presented herein may be used for design purposes within the stated limitations of the equations.

\section{References}

[1] Nadai, A., Die Verbiegungen in einzelnen Punkten understützten kriesförmiger Platten, Physik. Z. 23, 366-376 (1922).

[2] Bassali, W. A., The Transverse Flexure of Thin Elastic Plates Supported at Several Points, Proceedings Cambridge Philosophical Society, 53, 728-743 (1957).

[3] Muskhelishvili, N. I., Some Basic Problems of the Mathematical Theory of Elasticity, 3d edition (Moscow, 1949).

[4] Timoshenko, S. and Woinowsky-Krieger, S., Theory of Plates and Shells, 2d ed. (McGraw-Hill Book Company, New York, N.Y., 1959).

[5] Dawoud, R. H., Continuity and Boundary Conditions in Thin Elastic Plates, Proceedings of the Mathematical and Physical Society of Egypt, 5, 51-57, 1954.

[6] Abramowitz, M., and Stegun, I. A., (editors), Handbook of Mathematical Functions, National Bureau of Standards, AMS Series No. 55, (1964).

[7] Lewin, L., Dilogarithms and Associated Functions (Macdonald, London, England, 1958).

[8] Mitchell, K., Tables of the Function $\int_{0}^{z} \frac{\log |1-y|}{y} d y$, with an Account of Some Properties of This and Related Functions, Phil. Mag. No. 40, 351-368 (1949).

(Paper 70C4-234) 